\title{
Research on the distribution spectrum of atherosclerotic plaques in patients with suspected coronary artery disease and the noninvasive screening model for coronary atherosclerosis burden
}

\author{
Pengxi Han ${ }^{1}$, Jinyan Tang ${ }^{2}$, Ximing Wang ${ }^{3}$, Yuwen $\mathrm{Su}^{1}$, Guijie $\mathrm{Li}^{4 \#}$, Kai Deng ${ }^{1 \#}$ \\ ${ }^{1}$ Department of Radiology, the First Affiliated Hospital of Shandong First Medical University, Jinan, China; ${ }^{2}$ Department of Pediatrics, Xiangxi \\ Autonomous Prefecture People's Hospital, Jishou, China; ${ }^{3}$ Department of Radiology, Shandong Provincial Hospital of Shandong First Medical \\ University, Jinan, China; ${ }^{4}$ Department of Interventional Radiology, the First Affiliated Hospital of Shandong First Medical University, Jinan, China
}

\#These authors contributed equally to this work.

Correspondence to: Kai Deng. Department of Radiology, the First Affiliated Hospital of Shandong First Medical University, 16766 Jingshi Road, Jinan 250014, China. Email: 289954749@qq.com; Guijie Li. Department of Interventional Radiology, the First Affiliated Hospital of Shandong First Medical University,16766 Jingshi Road, Jinan 250014, China. Email: Lilijie-2450@163.com.

Background: This study aimed to establish a non-invasive and simple screening model of coronary atherosclerosis burden based on the associations between multiple blood parameters and total plaque score (TPS), segment-stenosis score (SSS), coronary artery disease severity (CADS) in coronary artery disease (CAD) and thus reduce unnecessary coronary angiography (CAG).

Methods: A total of 1,366 patients with suspected CAD who underwent CAG were included in this study. The clinical risk factors [age, gender, systolic blood pressure (SBP), diastolic blood pressure (DBP), total cholesterol (TC), high-density lipoprotein (HDL), triglyceride (TG), low-density lipoprotein (LDL), fasting plasma glucose (FPG), and glycated hemoglobin (GHB)] were collected. The presence of plaques and lumen stenosis was assessed based on CAG imaging. The TPS, SSS, and CADS were calculated, and the distribution spectrum of atherosclerotic plaques was described. Kruskal-Wallis test for multiple comparison tests was performed to analyze the differences in groups of different risk factors. The selected independent predictors were put into a multivariate logistic model, and the variables were further screened by stepwise regression to establish a screening model. Finally, the receiver operating characteristic (ROC) curve was used to evaluate the selected model's discriminant effect.

Results: The distributions of TPS and SSS scores were both right-skewed. Among males, both TPS and SSS scores were higher than in females $\left(\chi^{2}=46.7659, \mathrm{P}<0.0001, \chi^{2}=51.6603, \mathrm{P}<0.0001\right)$. Both TPS and SSS scores increased with age $\left(\chi^{2}=123.4456, \mathrm{P}<0.0001, \chi^{2}=123.4456, \mathrm{P}<0.0001\right)$. For TPS, the most common position was proximal left anterior descending artery (P-LAD, 51.39\%). In SSS, the P-LAD plaque was highest: $0: 48.61 \%, 1: 10.32 \%, 2: 9.15 \%$, and $3: 31.92 \%$. The TPS score $>5$, SSS score $>5$, and CAD $>0$ were valuable indicators for SBP, FPG, TG, HDL, and GHB. In the model, TPS score $>5$, SSS score $>5$, and CADS $>0$, the area under ROC curve (AUC) was 0.753 [95\% confidence interval (CI): 0.713 to 0.789 ], 0.728 (95\% CI: 0.687 to 0.766 ), and 0.756 (95\% CI: 0.717 to 0.793 ), respectively.

Conclusions: The most common site of lesions was P-LAD. These models can be used as non-invasive and simple initial screening tools without CAG.

Keywords: Coronary artery disease (CAD); plaque distribution spectrum; non-invasive screening model; coronary angiography (CAG)

Submitted Jul 22, 2020. Accepted for publication Mar 31, 2021.

doi: 10.21037/qims-20-901

View this article at: http://dx.doi.org/10.21037/qims-20-901 


\section{Introduction}

Coronary artery disease (CAD) is a common disease. According to the Global Atlas on Cardiovascular Disease Prevention and Control published by the WHO in 2011, $\mathrm{CAD}$ surpassed cancer and became the biggest cause of death worldwide (1). It has become a chronic disease with the highest incidence, mortality, and disability rate in China (2). Therefore, improving the early diagnosis of coronary heart disease (CHD) is particularly important for early treatment, minimizing myocardial damage, and improving survival and prognosis.

Coronary angiography (CAG) is currently considered the "gold standard" for diagnosing CAD. Presently, CAG is an important method to determine the location and severity of coronary lesions and accurately treat them, and obtain a positive prognosis. However, not all patients with suspected CAD require $\mathrm{CAG}(3,4)$.

From the early occurrence and development of CAD to the late transition to serious adverse events, many risk factors accumulate, resulting in the interaction, continuous stimulation, and damage of the vascular endothelial system (5). There are many pathogenic factors of CAD, among which the main factors have been very clear; age, gender, hypertension, smoking, diabetes, and so on. Many studies at home and abroad have shown that CHD has traditional risk factors, and about $85-90 \%$ of CHD patients have traditional risk factors (6). Therefore, it is very necessary to establish a risk assessment model of CAD according to the risk factors to assess the necessity of CAG.

At present, for the risk assessment of CAD, the most prominent model proposed by international clinical guidelines is the Framingham CAD risk assessment (FHS), pooled cohort equation $(6,7)$. However, there are some problems in these models: (I) they are based on European and American people; because of the differences of exposure levels and socio-economic and cultural backgrounds in different regions and varied population characteristics, these models may not be inappropriate for use among Chinese demographics $(8,9)$. (II) The base of reference of these models was community populations, and for patients with suspected CHD, there may be additional problems that remain unaccounted for in the models.

This study aimed to establish a non-invasive and simple screening model for coronary atherosclerosis burden based on relevant risk factors in Chinese patients with suspected CAD.

\section{Methods}

\section{Research population}

Between March 2016 and November 2019, in the First Affiliated Hospital of Shandong First Medical University, a total of 1,366 patients who underwent CAG were enrolled concerning the inclusion and exclusion criteria.

The inclusion criteria were as follows: patients with suspected CAD who showed related symptoms (chest tightness, palpitations, chest pain after exertion, and so on) at their first hospital visit; clinical history was provided with detailed and complete data; patients had not undergone CAG, interventional therapy, or had coronary artery bypass grafting before this CAG. Patients with allergies to contrast media, severe systemic infections, severe heart, lung, liver, or renal insufficiency, and systemic immune diseases were excluded. Previous studies had clearly shown that CAD is closely related to smoking and weight, factors that may have greatly impacted our research's specificity and sensitivity; therefore, patients with a BMI $\leq$ of 25 or who had been smoking for more than 5 years were excluded.

\section{Research methods}

\section{Risk factors}

All diagnoses of diabetes, hypertension, and dyslipidemia were based on the WHO.. Diabetes was defined as fasting plasma glucose (FPG) $\geq 70 \mathrm{mmol} / \mathrm{L}$, and/or 2 hour postprandial glucose or random blood glucose $\geq 11.1 \mathrm{mmol} / \mathrm{L}$, or a definite history of diabetes. Hypertension was defined as systolic blood pressure (SBP) $\geq 140 \mathrm{mmHg}$, and/or diastolic blood pressure (DBP) $\geq 90 \mathrm{mmHg}$, or a definite history of hypertension. The criteria for hyperlipidemia included total cholesterol (TC) $\geq 5.18 \mathrm{mmol} / \mathrm{L}$, low-density lipoprotein cholesterol (LDL-C) $\geq 3.37 \mathrm{mmol} / \mathrm{L}$; the criteria for hypolipidemia included high-density lipoprotein cholesterol (HDL-C) $\leq 1.04 \mathrm{mmol} / \mathrm{L}$, triglyceride (TG) $\geq 1.7 \mathrm{mmol} / \mathrm{L}(7-9)$.

\section{CAG}

The CAG was performed by a cardiologist and routinely via the radial artery. In case of unsuccessful puncture or vasospasm, femoral artery puncture was the alternative. According to the American Heart Association (AHA), basic angiographic views included left anterior oblique position, spider view, anteroposterior position plus caudal position, 

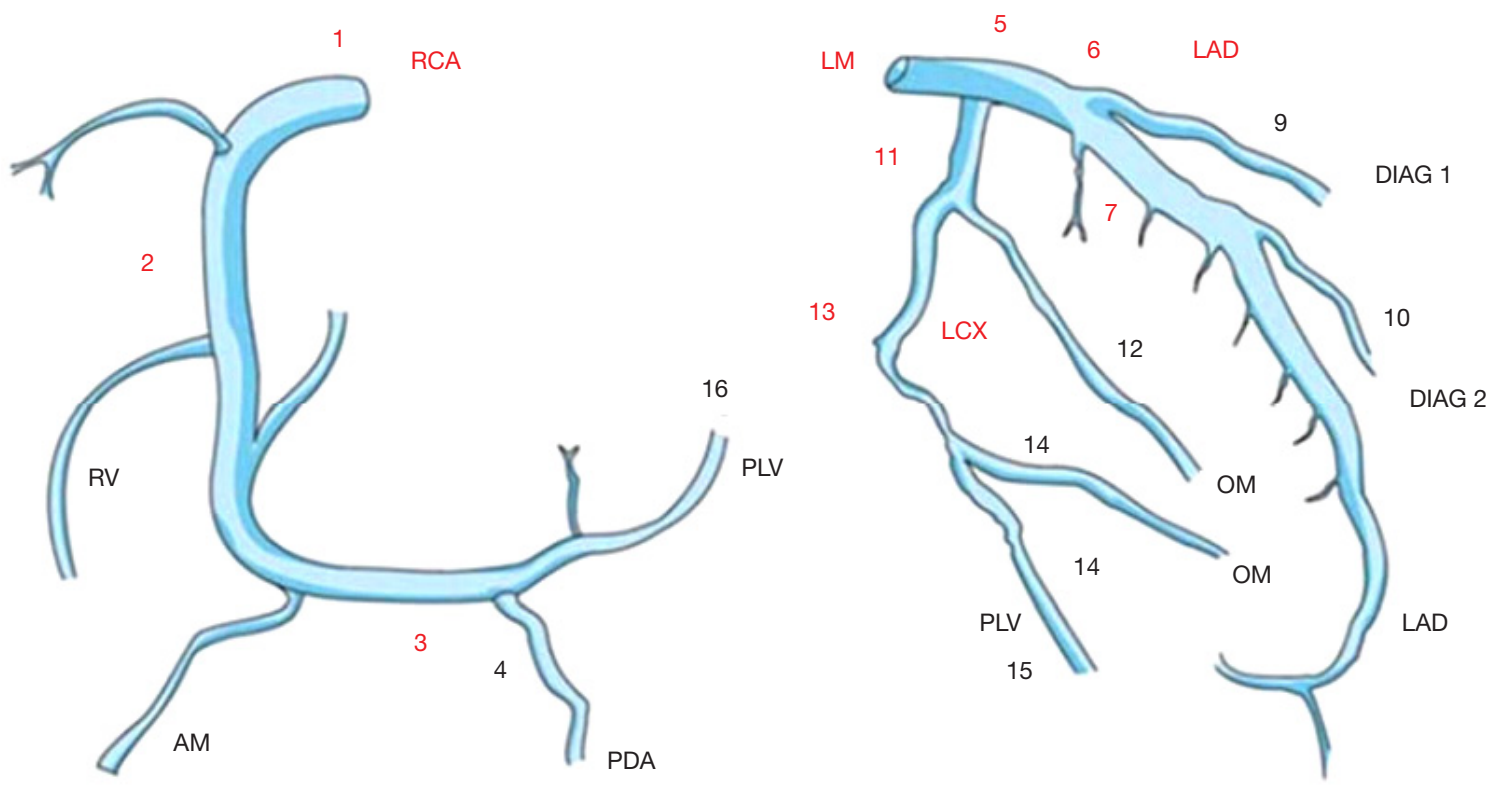

Figure 1 Anatomy and segmentation of the coronary artery. RCA, right coronary artery; RV, right ventricular branch; AM, acute marginal branch; PDA, posterior descending artery; PLV, posterior left ventricular branch; LM, left main; LAD, left anterior descending; DIAG, diagonal branch; OM, obtuse marginal; LCX, left of circumflex.

and right anterior oblique position for left coronary artery; left anterior oblique position, left anterior oblique position plus cranial position, and right anterior oblique position for right coronary artery. Additional positions were able to be adopted if necessary.

\section{Image analysis}

\section{6-segment model of coronary artery (Figure 1)}

According to the detailed classification rules of the coronary artery system in the AHA (9), the coronary arteries was divided into 16 segments.

\section{Assessment of coronary artery stenosis}

According to the degree of coronary artery stenosis, coronary arteries were often divided into 4 groups: normal; mild lesions, stenosis less than 50\%; moderate lesions, stenosis of $50-69 \%$; severe lesions, stenosis greater than $70 \%(10,11)$.

\section{Assessment of coronary atherosclerotic plaques}

Each coronary segment in the angiogram was analyzed, and the presence of atherosclerotic plaques was determined by the degree of vascular stenosis, intima-media thickness, and hemodynamics (12).

\section{Scores}

By analyzing the results of coronary atherosclerotic plaques, total plaque score (TPS), segment-stenosis score (SSS), and coronary artery disease severity (CADS) were calculated to quantitatively evaluate coronary plaque burden and describe the distribution spectrum of coronary atherosclerotic plaques in detail (12). The TPS was calculated by summing the number of atherosclerotic plaques, regardless of hemodynamic changes or vascular stenosis. The maximum score of patients with suspected CHD was $16(12,13)$. Calculation of the SSS required detailed evaluation and analysis of stenosis degree in each coronary segment (normal, 0 points; mild lesions with stenosis less than 50\%, 1 point; moderate lesions with stenosis of $50-69 \%, 2$ points; severe lesions with stenosis greater than $70 \%, 3$ points). The SSS was calculated by summation of stenosis degree scores of 16 segments, with a maximum score of 48 (13). The CADS was mainly focused on whether the coronary artery's stenosis severity had caused hemodynamic changes and whether it could affect the patient's myocardial blood supply. Participants were divided into 3 categories according to the stenosis degree of the most severe lesion inside the coronary artery, namely normal ( 0 , no stenosis or plaque); non-occlusive coronary artery disease $(1$, stenosis $\leq 50 \%$ 
and plaque formation); occlusive coronary artery disease (2, stenosis $>50 \%$ ) (14). Image analysis was performed via a subjective method by 2 radiologists (with 10 years of experience); if there was a discrepancy between results, the 2 radiologists reached consensus through discussion or decided by a third radiologist (with 15 years of experience).

\section{Detection of risk factors}

All participants were asked for their detailed medical history before CAG, and blood pressure was measured daily. Various factors (TG, TC, HDL-C, LDL-C, and $\mathrm{GHB}$ ) were detected with fasting blood by an automatic biochemical analyzer (7-9).

\section{Data analysis}

We found that the TPS, SSS, and CADS of participants were non-normally distributed. Thus, the mean and variance of TPS, SSS, and CADS were described by mean \pm standard deviation $(\mathrm{SD})$ and median \pm interquartile range (IQR). The H-test for multiple comparative tests was performed to analyze further the differences between groups (gender groups and age groups). To visually describe the burden of coronary atherosclerotic plaque, the incidence of plaque in each coronary segment and different stenosis levels were marked on a tree diagram of the coronary artery.

To determine the independent predictors of coronary stenosis, a univariate logistic model adjusted for age and gender was used for screening with $\alpha=0.05$ set as the statistical significance level. The model was as follows.

$$
P=\frac{\exp \left(\beta_{0}+\beta_{\text {age }} \text { age }+\beta_{\text {gender }} \text { gender }+\beta_{i} X_{i}\right)}{1+\exp \left(\beta_{0}+\beta_{\text {age }} \text { gege }+\beta_{\text {gender }} \text { gender }+\beta_{i} X_{i}\right)}
$$

The statistically significant predictors selected by a univariate logistic model adjusted for age and gender were entered into a multivariate logistic model to determine the screening model. The variables were further screened by stepwise regression to establish a screening model for patients with suspected CHD finally. The model was as follows.

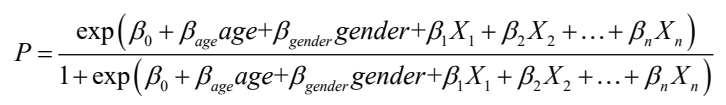

The dichotomy of TPS or SSS $>5$ versus TPS or SSS $\leq 5$ was used to divide the participants into 2 groups, defined value as 1 and 0 , respectively (11).

For CADS, a participant's defined value with non- occlusive coronary artery disease or occlusive coronary artery disease was 1 , while the suspected patient without plaques or stenosis was 0 . The assignment of other independent variables is shown in the following table (Table 1). All of the analyses mentioned above-containing hypothesis tests were considered statistically significant with $P$ values $<0.05$. Statistical analyses were performed with SAS 9.4 statistical software (SAS Institute Inc., Cary, NC, USA).

In this study, the screened model's discriminant effect was evaluated by a receiver operating characteristic (ROC) curve and the area under the ROC curve (AUC). After drawing the ROC curve and marking the optimum cutoff value on the curve, the AUC and its $95 \%$ confidence interval (CI) were calculated. Different cut-off values for higher sensitivity and specificity were also obtained. Plotting of ROC curve and calculation of AUC, sensitivity, and specificity in this study were performed by using MedCalc (MedCalc software Ltd., Ostend, Belgium).

\section{Results}

\section{Participant characteristics}

Of the 1,366 participants who underwent CAG, 261 had no stenosis, and 1,105 had stenosis. The mean age of the nonstenotic population was $55.60 \pm 9.36$, and the mean age of the stenotic population was $60.80 \pm 10.6$; the difference was statistically significant $(\mathrm{P}<0.01)$.

As compared to participants without stenosis, those with stenosis had higher levels of blood pressure, FPG, TC, and GHB, and lower HDL-cholesterol levels. The differences were statistically significant except for TG and LDLcholesterol (Table 1).

\section{The distribution of coronary atherosclerosis burden}

Both the TPS and SSS displayed a skewed distribution with a significant long tail on the right. The overall trend was shown that the number of participants with low SSS was higher than that of those with high SSS. High scores accounted for a relatively low percentage, and most of the scores were concentrated on relatively low levels. Among participants, $7.17 \%(98 / 1,366)$ had SSS $>20$ and $5.56 \%$ $(76 / 1,366)$ had TPS $>8 ; 44.36 \%(606 / 1,366)$ had SSS $\leq 5$ and $76.57 \%(1,046 / 1,366)$ had TPS $\leq 5$ (Figures 2,3$)$.

Table 2 shows that there were significant differences in TPS and SSS between gender and different age groups. For TPS, the mean value of males $(2.81 \pm 2.81)$ was higher 
Table 1 Comparison between stenotic group and non-stenosis group

\begin{tabular}{lccc}
\hline Index & Non-stenosis $(\mathrm{n}=261)$ & Stenosis $(\mathrm{n}=1,105)$ & $\mathrm{P}$ value \\
\hline Age & $55.60 \pm 9.36$ & $60.80 \pm 10.63$ & $<3.0001$ \\
SBP & $141.23 \pm 26.75$ & $150.25 \pm 29.97$ & $<0.0001$ \\
DBP & $84.85 \pm 15.54$ & $88.83 \pm 18.80$ & 0.0004 \\
FPG $(\mathrm{mmol} / \mathrm{L})$ & $5.45 \pm 1.39$ & $6.00 \pm 2.00$ & $<0.0001$ \\
TG $(\mathrm{mmol} / \mathrm{L})$ & $1.48 \pm 1.43$ & $1.58 \pm 1.05$ & 0.3053 \\
TC $(\mathrm{mmol} / \mathrm{L})$ & $4.56 \pm 1.04$ & $4.38 \pm 1.11$ & 0.0177 \\
HDL-C (mmol/L) & $1.30 \pm 0.35$ & $1.16 \pm 0.36$ & $<0.0001$ \\
\hline
\end{tabular}

SBP, systolic blood pressure, DBP, diastolic blood pressure, FPG, fasting plasma glucose; TG, triglycerides; TC, total cholesterol; HDL-C, high-density lipoprotein; LDL-C, low-density lipoprotein.S

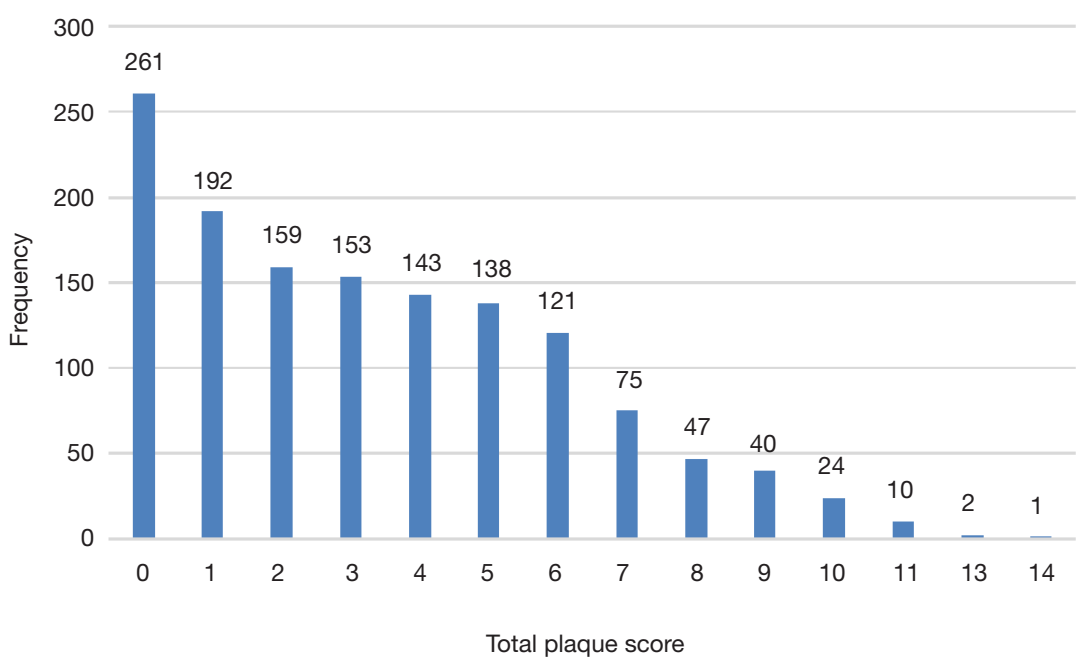

Figure 2 Frequency distribution of TPS in the population. TPS, total plaque score.

than that of females $(3.75 \pm 2.76)(\mathrm{P}<0.0001)$, and female SSS $(6.60 \pm 7.20)$ was lower than that of males $(9.11 \pm 7.24)$ $(\mathrm{P}<0.0001)$. Among different age groups, the mean value of TPS for participants aged $<52$ was $2.45 \pm 2.52$, aged $52-59$ was $2.85 \pm 2.69$, aged $60-67$ was $3.51 \pm 2.86$, and aged $>68$ was $4.60 \pm 2.69$; the difference was statistically significant $(\mathrm{P}<0.0001)$. The same trend was shown among participants for SSS $(\mathrm{P}<0.0001)$.

\section{The distribution of coronary plaque burden in different vascular segments}

For TPS, the highest incidence was $51.39 \%(701 / 1,366)$ in the anterior descending branch's proximal segment. The incidence of TPS was $39.68 \%(542 / 1,366)$ in the middle segment of the anterior descending branch, $31.55 \%$ $(431 / 1,366)$ in the proximal segment of the right coronary artery, $28.92 \%(395 / 1,366)$ in the middle segment of the right coronary artery, and $27.89 \%(381 / 1,366)$ in the proximal segment of the levolateral branch (Figure 4).

For SSS, the proximal segment of the anterior descending branch had the highest frequency of plaques (SSS distribution was 0: $48.61 \%, 1: 10.32 \%, 2: 9.15 \%$, $3: 31.92 \%)$, followed by mid segment of the anterior descending branch $(0: 60.32 \%, 1: 7.1 \%, 2: 8.86 \%, 3$ : $23.72 \%$ ), proximal segment of the right coronary artery (0: $68.45 \%, 1: 8.64 \%, 2: 5.93 \%, 3: 16.98 \%$ ), mid segment of the right coronary artery $(0: 71.08 \%, 1: 7.54 \%, 2: 5.49 \%, 3$ : $15.89 \%)$, and proximal segment of the left circumflex branch (0: 72.11\%, 1: 7.03\%, 2: 6.3\%, 3: 14.57\%) (Figure 5). 


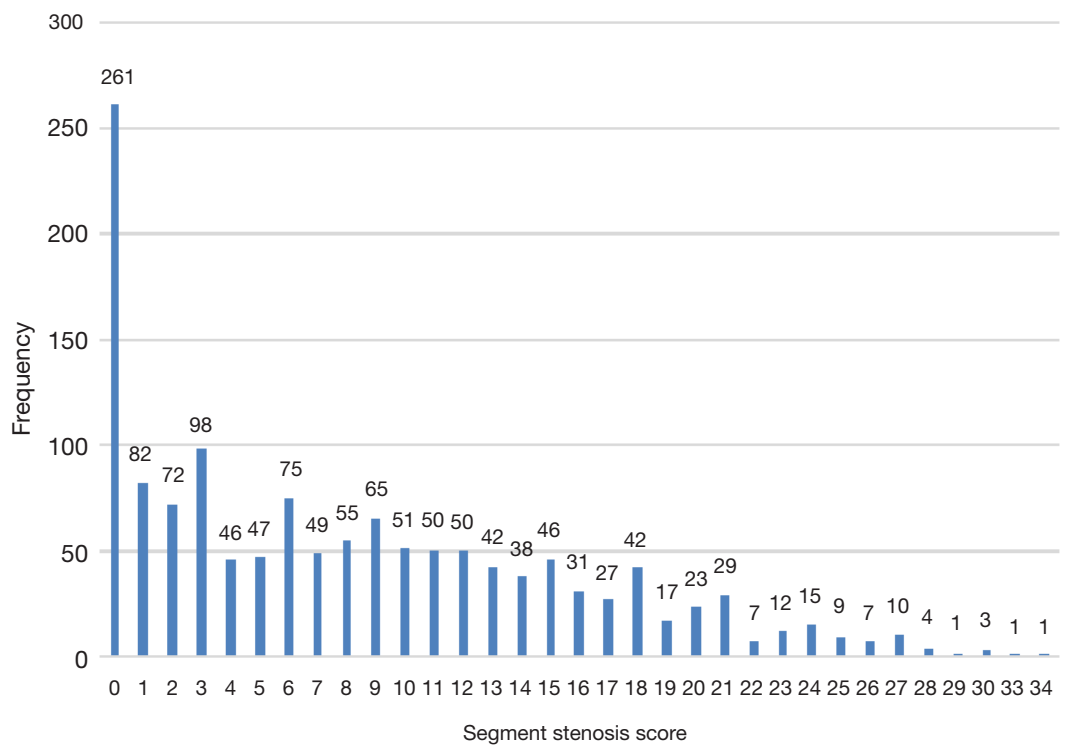

Figure 3 Frequency distribution of SSS in the population. SSS, segment-stenosis score.

Table 2 Comparison of TPS and SSS between genders and different age groups

\begin{tabular}{|c|c|c|c|c|c|c|c|c|}
\hline Groups & $\mathrm{n}$ & \multicolumn{2}{|c|}{ TPS } & \multicolumn{2}{|c|}{ SSS } & \multicolumn{3}{|c|}{ CADS, n (\%) } \\
\hline \multicolumn{9}{|l|}{ Gender } \\
\hline Female & 53 & $2.81 \pm 2.81$ & $2 \pm 2$ & $6.60 \pm 7.20$ & $4 \pm 5.5$ & $150(27.12)$ & 105 (18.99) & 298 (53.89) \\
\hline Male & 13 & $3.75 \pm 2.76$ & $3 \pm 2.5$ & $9.11 \pm 7.24$ & $8 \pm 5.5$ & $111(13.65)$ & 95 (11.69) & $607(74.66)$ \\
\hline \multicolumn{9}{|c|}{ Age, years } \\
\hline$<52$ & 9 & $2.45 \pm 2.52$ & $2 \pm 2$ & $5.73 \pm 6.23$ & $4 \pm 4.5$ & $86(27.83)$ & 42 (13.59) & 181 (58.58) \\
\hline $52-59$ & 65 & $2.85 \pm 2.69$ & $2 \pm 2.5$ & $6.64 \pm 6.90$ & $4 \pm 5.5$ & $92(25.21)$ & $71(19.45)$ & 202 (55.34) \\
\hline $60-67$ & 43 & $3.51 \pm 2.86$ & $3 \pm 2$ & $8.43 \pm 7.56$ & $7 \pm 5.5$ & 56 (16.33) & $51(14.87)$ & $236(68.8)$ \\
\hline
\end{tabular}

SSS, segment-stenosis score; TPS, total plaque score; CDS, coronary artery disease severity.

\section{Relationship between clinical indexes and scores of atherosclerotic plaque burden}

Values in this analysis were defined as 1 when TPS $>5$, SSS $>5$, or CADS indicating stenosis, otherwise values were defined as 0 . The TPS could be predicted by SBP [odds ratio (OR): 1.008 (95\% CI: 1.004 to 1.013)], FPG [OR: 1.232 (95\% CI: 1.156 to 1.316$)$ ], TG [OR: 1.171 (95\% CI: 1.051 to 1.311 )], HDL-C [OR: 0.442 (95\% CI: 0.267 to
0.708)], GHB [OR: 1.454 (95\% CI: 1.292 to 1.643$)$ ]. The SSS could be predicted by SBP [OR: 1.010 (95\% CI: 1.006 to 1.015 )], DBP [OR: 1.011 (95\% CI: 1.005 to 1.018 )], FPG [OR: 1.236 (95\% CI: 1.148 to 1.339$)$ ], TG [OR: 1.216 (95\% CI: 1.082 to 1.379 )], HDL-C [OR: 0.427 (95\% CI: 0.272 to 0.653)], GHB [OR: 1.390 (95\% CI: 1.218 to 1.600$)$ ]. The CADS could be predicted by SBP [OR: 1.012 (95\% CI: 1.007 to 1.017)], DBP [OR: 1.017 (95\% CI: 1.009 to 1.026)], FPG [OR: 1.201 (95\% CI: 1.087 to 1.344$)$ ], HDL-C [OR: 

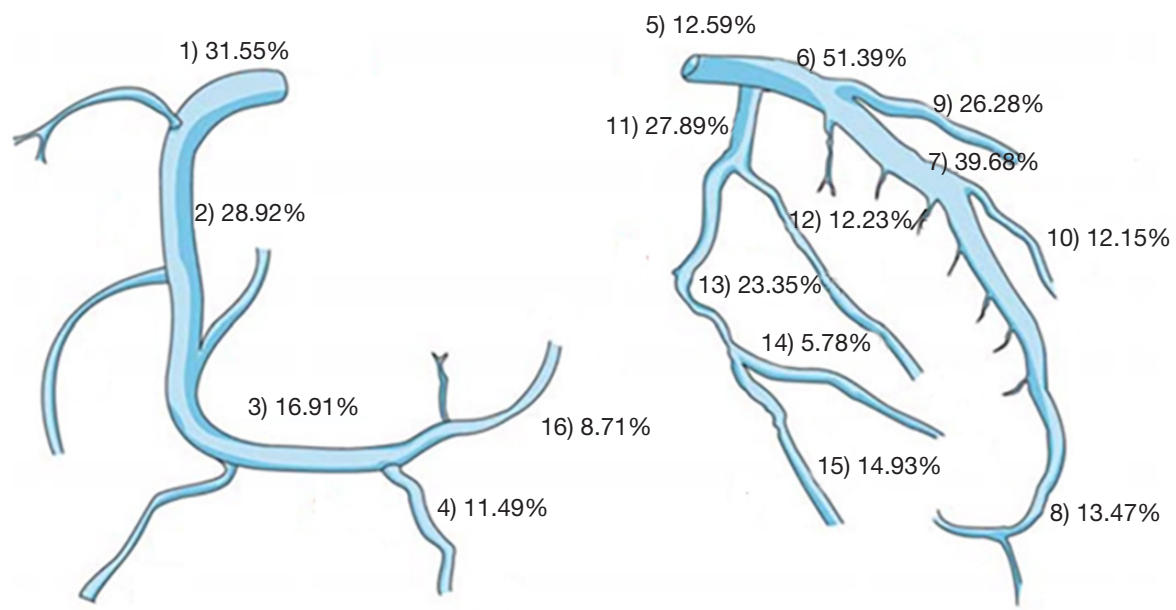

Figure 4 Coronary plaque burden TPS of different vascular segments showed in the tree diagram of coronary artery. TPS, total plaque score.
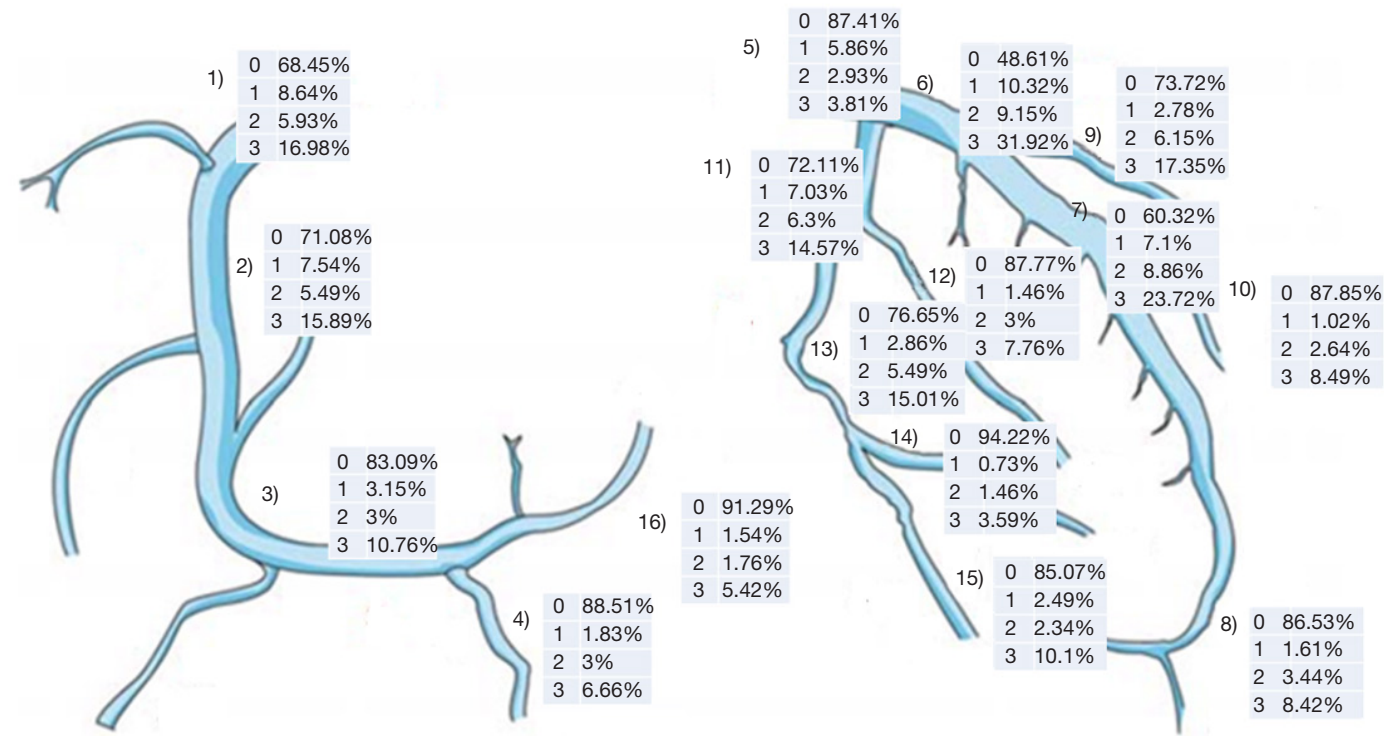

Figure 5 Coronary plaque burden SSS of different vascular segments showed in the tree diagram of coronary artery. SSS, segment-stenosis score.

0.492 (95\% CI: 0.310 to 0.776$)$ ], and GHB [OR: $1.511(95 \%$ CI: 1.245 to 1.885 )] (Table 3).

\section{Screening model}

The statistically significant predictors selected by a logistic model adjusted for age and gender were entered into the screening model, and the variables were further screened by stepwise regression. A screening model of CADS $>0, \mathrm{SSS}>5$, or TPS $>5(8,9,11)$ could be established as follows (Table 4).

Table 5 and Figure 6 show the sensitivity and specificity under different cut-off values in the 3 models. In CADS >0 model, the AUC was 0.753 (95\% CI: 0.713 to 0.789 ) and the optimum cut-off value was 0.6114 with a sensitivity and specificity of $74.2 \%$ and $59.1 \%$, respectively (Figure $6 A$ ). When the cut-off value was $0.5665,0.4876,0.3801$, and 0.3491 , the sensitivity became $80 \%, 90 \%, 95 \%$, and $97.5 \%$, respectively. 
Table 3 Results of logistic regression analysis for scores of atherosclerotic plaque burden through clinical indexes adjusted for gender and age

\begin{tabular}{lccc}
\hline Risk factors & TPS, OR $(95 \% \mathrm{Cl})$ & SSS, OR $(95 \% \mathrm{Cl})$ & CADS, OR (95\% CI) \\
\hline SBP & $1.008(1.004,1.013)^{\star}$ & $1.010(1.006,1.015)^{\star}$ & $1.012(1.007,1.017)^{\star}$ \\
DBP & $1.006(0.999,1.013)$ & $1.011(1.005,1.018)^{\star}$ & $1.017(1.009,1.026)^{\star}$ \\
FPG $(\mathrm{mmol} / \mathrm{L})$ & $1.232(1.156,1.316)^{\star}$ & $1.236(1.148,1.339)^{\star}$ & $1.201(1.087,1.344)^{\star}$ \\
TG $(\mathrm{mmol} / \mathrm{L})$ & $1.171(1.051,1.311)^{\star}$ & $1.216(1.082,1.379)^{\star}$ & $1.148(0.995,1.347)$ \\
TC $(\mathrm{mmol} / \mathrm{L})$ & $1.031(0.919,1.156)$ & $0.991(0.889,1.106)$ & $0.934(0.819,1.067)$ \\
HDL-C (mmol/L) & $0.442(0.267,0.708)^{\star}$ & $0.427(0.272,0.653)^{\star}$ & $0.492(0.310,0.776)^{\star}$ \\
LDL-C (mmol/L) & $1.115(0.967,1.284)$ & $1.050(0.916,1.206)$ & $0.996(0.843,1.180)$ \\
GHB $(\%)$ & $1.454(1.292,1.643)^{\star}$ & $1.390(1.218,1.600)^{\star}$ & $1.511(1.245,1.885)^{\star}$ \\
\hline
\end{tabular}

*, $\mathrm{P}<0.05$. SSS, segment-stenosis score; TPS, total plaque score; CADS, coronary artery disease severity; SBP, systolic blood pressure; DBP, diastolic blood pressure; FPG, fasting plasma glucose; TG, triglycerides; TC, total cholesterol; HDL-C, high-density lipoprotein; LDL-C, low-density lipoprotein; GBH, glycated hemoglobin.

Table 4 Screening model of CADS $>0$, SSS $>5$, or TPS $>5$

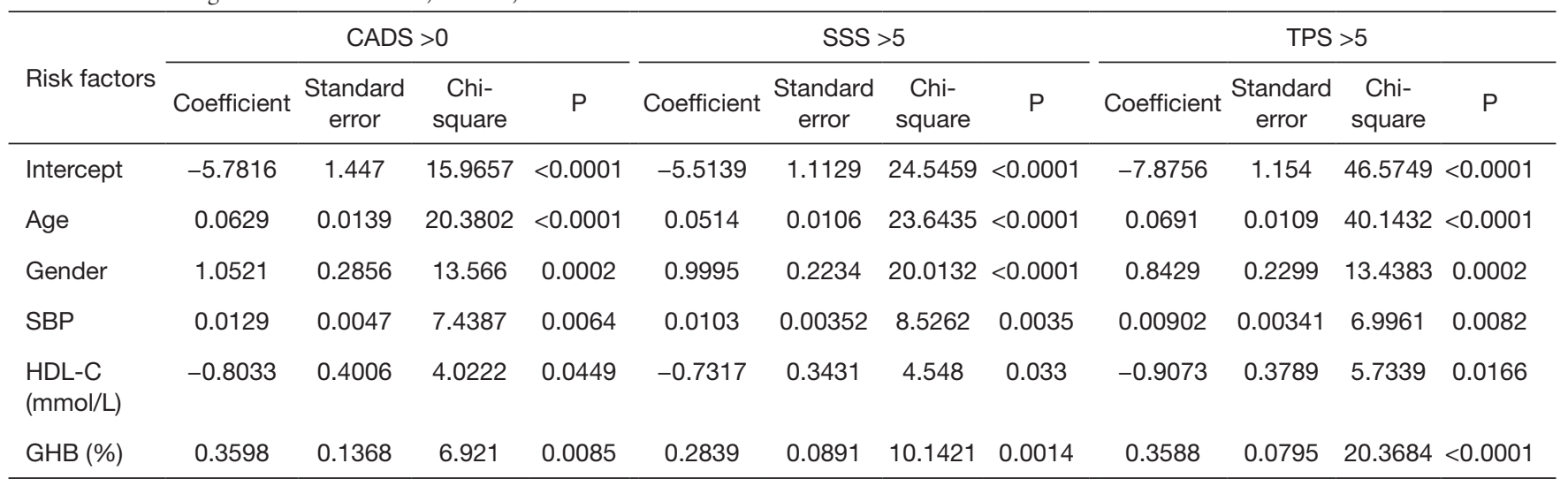

SBP, systolic blood pressure; HDL-C, high-density lipoprotein; GBH, glycated hemoglobin; CADS, coronary artery disease severity; SSS, segment-stenosis score; TPS, total plaque score; CADS, coronary artery disease severity.

For SSS $>5$, the AUC was 0.728 (95\% CI: 0.687 to 0.766$)$ and the cut-off value was 0.8909 , the sensitivity and specificity were $68.5 \%$ and $72.3 \%$, respectively (Figure $6 B$ ). When the cut-off value was $0.8476,0.7909,0.7412$, and 0.6921 , then the sensitivity became $80 \%, 90 \%, 95 \%$, and $97.5 \%$, respectively.

For TPS >5, the AUC was 0.756 (95\% CI: 0.717 to 0.793 ) and the optimum cut-off value was 0.3926 with a sensitivity and specificity of $66.5 \%$ and $73.8 \%$, respectively (Figure 6C). When the cut-off value was $0.3105,0.2238$, 0.1693 , and 0.1502 , the sensitivity became $80 \%, 90 \%, 95 \%$, and $97.5 \%$, respectively.

\section{Discussion}

This study showed that both TG and LDL were not significantly different between stenotic and non-stenotic groups (Table 1), which was different from previous studies $(8,9)$. We postulated that it might have been related to the difference in the numbers of stenotic and nonstenotic groups in this study. Moreover, patients who have undergone CAG examination are suspected of having CAD; they may be in the early stage of CAD when the plaques have not yet caused stenosis. Therefore, blood parameters such as LDL and TG were not statistically different between participants with and without coronary stenosis.

This study also revealed a negative correlation between HDL and CAD incidence, which was consistent with several epidemiological studies (14-16). Theoretically, HDL's main physiological function is to reverse cholesterol transport by transporting cholesterol from peripheral tissues 
Table 5 The sensitivity and specificity of the three models (CADS $>0$, SSS $>5$, TPS $>5$ ) under different cut-off values

\begin{tabular}{|c|c|c|c|c|}
\hline Valuable indicators & Sensitivity (\%) & Specificity (\%) & $95 \% \mathrm{Cl}$ & Cut-off value \\
\hline \multirow{3}{*}{ CADS $>0$} & 90 & 39.25 & $30.84-50.78$ & $>0.7909$ \\
\hline & 95 & 28.04 & 19.31-37.99 & $>0.7412$ \\
\hline & 97.5 & 21.81 & $11.53-30.53$ & $>0.6921$ \\
\hline \multirow{3}{*}{ SSS $>5$} & 90 & 38.12 & $24.31-48.62$ & $>0.4876$ \\
\hline & 95 & 20.99 & $14.36-27.62$ & $>0.3801$ \\
\hline & 97.5 & 18.23 & $9.39-24.86$ & $>0.3491$ \\
\hline \multirow[t]{2}{*}{ TPS $>5$} & 80 & 58.88 & $49.84-68.69$ & $>0.3105$ \\
\hline & 97.5 & 21.18 & $11.53-28.04$ & $>0.1502$ \\
\hline
\end{tabular}

CADS, coronary artery disease severity; SSS, segment-stenosis score; TPS, total plaque score; CADS, coronary artery disease severity; $\mathrm{Cl}$, confidence interval.

A

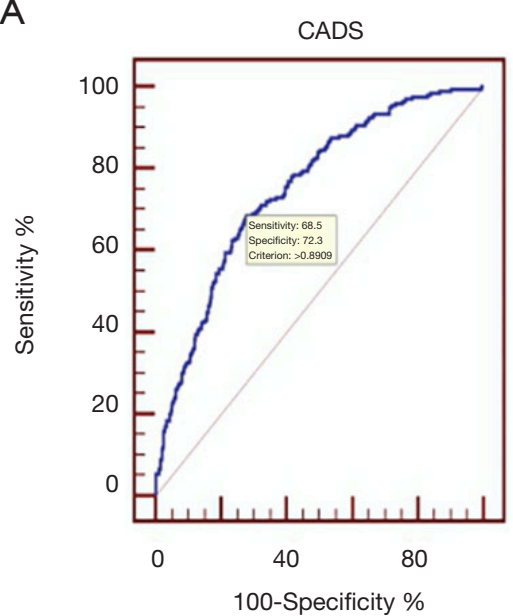

B

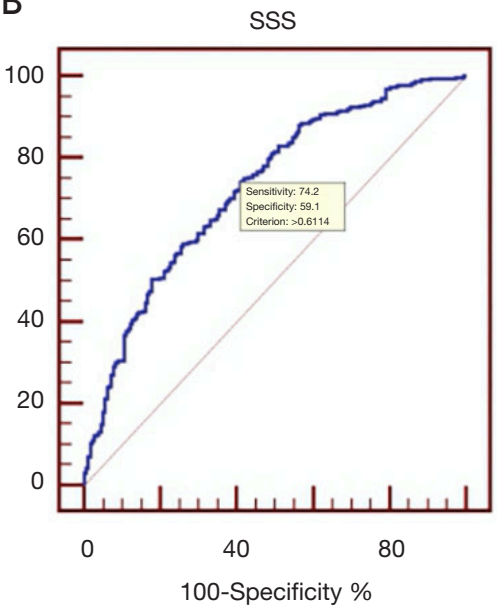

C

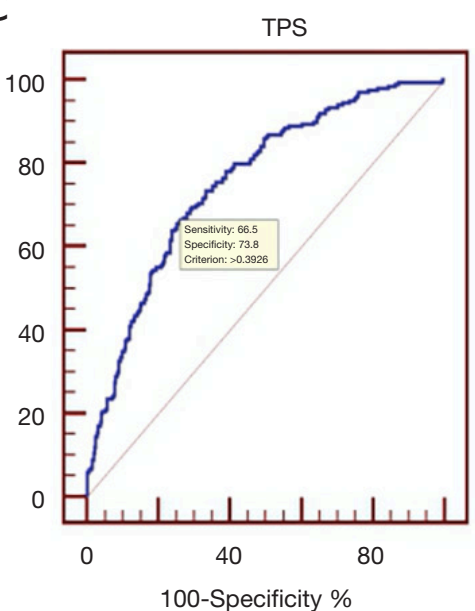

Figure 6 ROC of valuable indictors, sensitivity and specificity under different cut-off values. (A) CADS >0, AUC =0.753 (95\% CI: $0.713-0.789$ ), the optimum cut-off value is 0.6114. (B) SSS >5, AUC $=0.728$ (95\% CI: 0.687-0.766), the optimum cut-off value is 0.8909 . (C) TPS $>5$, AUC $=0.756$ (95\% CI: $0.717-0.793)$, the optimum cut-off value is 0.3926 . CADS, coronary artery disease severity; SSS, segment-stenosis score; TPS, total plaque score.

to the liver, from which point the cholesterol will be broken down and excreted as bile (16-18). This metabolic process is the basis for the prevention effect of HDL in CAD, and this theory can be used to explain the conclusions of this study.

Age is also an important risk factor for coronary stenosis, and the incidence of most cardiovascular diseases increases with age (either male or female) (Table 2). Framingham (9) in the United States also found that age is an important predictor of cardiovascular disease. Several basic studies on the pathology of coronary atherosclerosis found that atherosclerotic plaques' formation and development gradually progressed over time. Both lipid deposition on the vessel wall and smooth muscle migration is closely related to time, and the occurrence has shown a significant time dependence. As age increases, changes in cells, enzymes, molecules, and other substances in the vascular wall will cause reduced vascular 
elastic fibers, increased fibronectin, and calcification, further affecting vascular endothelium's function, leading to the formation of coronary atherosclerotic plaque $(19,20)$.

Moreover, in the results of this study, the difference in TPS and SSS between different age groups was also statistically significant, and the older the age, the higher the score and the greater the degree of stenosis. Most studies have found that men are at high risk of developing $\mathrm{CAD}$, and the incidence in women is generally lower than that of men. However, the incidence of CAD in women significantly increases after menopause (21). In this study, the incidence of both TPS and SSS was found to be higher in men than in women. There were significant differences between genders: $\mathrm{P}<0.0001$ for TPS, $\mathrm{P}<0.0001$ for SSS, $\mathrm{P}<0.0001$ for CADS (Table 3). Similar gender differences can also be seen in the Taiwanese population (22), among whom a study also found that men were more likely to have coronary atherosclerosis than women and that the disease in men was more severe than that in women.

The distribution of coronary plaque burden in participants with suspected CAD is shown in Figures 4,5 by a tree diagram of the coronary artery. It can be seen that the most common site was the proximal segment of the anterior descending branch with an incidence of $51.39 \%$, followed by the mid-segment of the anterior descending branch $(39.68 \%)$ and proximal segment of the left circumflex branch $(27.89 \%)$. In general, the plaques tended to occur in the coronary artery's bifurcation and in the proximal segment. According to the Gensini scoring system for coronary artery lesions, the proximal segment of the left anterior descending artery (P-LAD) was found to have the highest scores, similar to the distribution of coronary atherosclerotic plaque burden $(6,7)$.

The risk factors selected by a univariate logistic model adjusted for age and gender were entered into a multivariate logistic model. The variables were further screened by stepwise regression to establish a screening model for patients with suspected coronary heart disease (Tables 4,5). Finally, a non-invasive screening model was established with age, gender, SBP, FPG, TC, HDL, and GHB as independent variables, and with TPS $>5$, SSS $>5$, and CADS $>0$ as response variables.

The prediction effects of all 3 models were good, indicating that these models could be used to predict coronary conditions in patients with suspected CHD without computed tomography angiography (CTA) or CAG. To a certain extent, this could eliminate patient reliance on CTA and CAG, which are invasive and/or could cause radiation damage, reducing unnecessary costs and saving medical resources. Using certain clinical indexes (age, gender, SBP, PG, TG, HDL, and GHB) as a simple surrogate indicator for coronary CTA and even CAG was feasible, and it could exert a good predictive role so that only qualified patients need to undergo CAG examination.

On the other hand, these 3 screening models also suggested the possibility of coronary stenosis to some extent so that early preventive healthcare could be taken to prevent any sudden changes before treatment and to avoid adverse consequences. Also, these models made the selection of treatment timing and treatment methods more purposeful, improving the prognosis of patients. The purpose of this model was to try to screen out potential coronary artery lesions in patients with suspected CHD and reduce the rate of misdiagnosis. Therefore, higher sensitivity is required, but the specificity requirement could be appropriately reduced. Changes in the cut-off values corresponding to higher sensitivity were listed in the results section. Results showed that higher sensitivity could be achieved by appropriately lowering the criteria to screen for more highrisk patients $(22,23)$.

\section{Limitations}

Due to the absence of a follow-up study of TPS and SSS in the Chinese population, we have had to follow the definition of high-risk individuals in the US population established by Min et al. (TPS >5; SSS $>5$ ). The definition of this high-risk individual is not necessarily applicable to the Chinese population. Cases used in this study were those with suspected CAD undergoing CAG, so that selection bias could not be avoided. It would be beneficial if further research was performed with increased diversity among the samples and conducted in a more representative population.

\section{Conclusions}

The tree-shaped graph of the coronary artery directly revealed the distribution of plaque burden in patients with CHD. The proximal LAD was the most common site of major involvement; next was proximal RCA, middle LAD, proximal LCX, and middle RCA.

Predictive outcomes of the 3 models were definitely valuable, and they have enabled prediction without CTA or CAG, which can enable non-invasive and simple screening and reduce the economic burden and missed rate of patients. 


\section{Acknowledgments}

Funding: None.

\section{Footnote}

Conflicts of Interest: All authors have completed the ICMJE uniform disclosure form (available at http://dx.doi. org/10.21037/qims-20-901). The authors have no conflicts of interest to declare.

Ethical Statement: This study has been approved by the ethics committee of the First Affiliated Hospital of Shandong First Medical University. This clinical study is a retrospective study, which only collects the clinical data of patients, does not interfere with the treatment plan of patients, and will not bring physiological risks to patients. Researchers try their best to protect the information provided by patients from disclosing personal privacy, so patients' informed consent can be exempted.

Open Access Statement: This is an Open Access article distributed in accordance with the Creative Commons Attribution-NonCommercial-NoDerivs 4.0 International License (CC BY-NC-ND 4.0), which permits the noncommercial replication and distribution of the article with the strict proviso that no changes or edits are made and the original work is properly cited (including links to both the formal publication through the relevant DOI and the license). See: https://creativecommons.org/licenses/by-nc-nd/4.0/.

\section{References}

1. Kim KJ, Choi SI, Lee MS, Kim JA, Chun EJ, Jeon $\mathrm{CH}$. The prevalence and characteristics of coronary atherosclerosis in asymptomatic subjects classified as low risk based on traditional risk stratification algorithm: assessment with coronary CT angiography. Heart 2013;99:1113-7.

2. Liu T, Yuan X, Wang C, Sun M, Jin S, Dai X. Quantification of plaque characteristics detected by dual source computed tomography angiography to predict myocardial ischemia as assessed by single photon emission computed tomography myocardial perfusion imaging. Quant Imaging Med Surg 2019;9:711-21.

3. Stone GW, Maehara A, Lansky AJ, de Bruyne B, Cristea E, Mintz GS, Mehran R, McPherson J, Farhat N, Marso SP, Parise H, Templin B, White R, Zhang Z, Serruys
PW. A prospective natural-history study of coronary atherosclerosis. N Engl J Med 2011;364:226-35.

4. Hanrahan MT, O'Flynn AM, McHugh SM, Kearney P, Kearney PM. Appropriate use of elective coronary angiography in patients with suspected stable coronary artery disease. Ir J Med Sci 2019;188:807-13.

5. Malakar AK, Choudhury D, Halder B, Paul P, Uddin A, Chakraborty S. A review on coronary artery disease, its risk factors, and therapeutics. J Cell Physiol 2019;234:16812-23.

6. Park KH, Ahn Y, Jeong MH, Chae SC, Hur SH, Kim YJ, Seong IW, Chae JK, Hong TJ, Cho MC, Bae JH, Rha SW, Jang YS. Different impact of diabetes mellitus on in-hospital and 1-year mortality in patients with acute myocardial infarction who underwent successful percutaneous coronary intervention: results from the Korean Acute Myocardial Infarction Registry. Korean J Intern Med 2012;27:180-8.

7. Liu J, Hong Y, D'Agostino RB Sr, Wu Z, Wang W, Sun J, Wilson PW, Kannel WB, Zhao D. Predictive value for the Chinese population of the Framingham CHD risk assessment tool compared with the Chinese MultiProvincial Cohort Study. JAMA 2004;291:2591-9.

8. Jespersen L, Hvelplund A, Abildstrøm SZ, Pedersen F, Galatius S, Madsen JK, Jørgensen E, Kelbæk H, Prescott E. Stable angina pectoris with no obstructive coronary artery disease is associated with increased risks of major adverse cardiovascular events. Eur Heart J 2012;33:734-44.

9. Goff DC Jr, Lloyd-Jones DM, Bennett G, Coady S, D'Agostino RB, Gibbons R, Greenland P, Lackland DT, Levy D, O’Donnell CJ, Robinson JG, Schwartz JS, Shero ST, Smith SC Jr, Sorlie P, Stone NJ, Wilson PW, Jordan HS, Nevo L, Wnek J, Anderson JL, Halperin JL, Albert NM, Bozkurt B, Brindis RG, Curtis LH, DeMets D, Hochman JS, Kovacs RJ, Ohman EM, Pressler SJ, Sellke FW, Shen WK, Smith SC Jr, Tomaselli GF; American College of Cardiology/American Heart Association Task Force on Practice Guidelines 2013 ACC/AHA guideline on the assessment of cardiovascular risk: a report of the American College of Cardiology/American Heart Association Task Force on Practice Guidelines. Circulation 2014;129:S49-73.

10. Plank F, Friedrich G, Dichtl W, Klauser A, Jaschke W, Franz WM, Feuchtner G. The diagnostic and prognostic value of coronary CT angiography in asymptomatic highrisk patients: a cohort study. Open Heart 2014;1:e000096.

11. Normann J, Mueller M, Biener M, Vafaie M, Katus HA, Giannitsis E. Effect of older age on diagnostic and prognostic performance of high-sensitivity troponin $\mathrm{T}$ 
in patients presenting to an emergency department. Am Heart J 2012;164:698-705.e4.

12. Lee JM, Doh JH, Nam CW, Shin ES, Koo BK. Functional Approach for Coronary Artery Disease: Filling the Gap Between Evidence and Practice. Korean Circ J 2018;48:179-90.

13. Huxley RR, Barzi F, Lam TH, Czernichow S, Fang X, Welborn T, Shaw J, Ueshima H, Zimmet P, Jee SH, Patel JV, Caterson I, Perkovic V, Woodward M. Isolated low levels of high-density lipoprotein cholesterol are associated with an increased risk of coronary heart disease: an individual participant data meta-analysis of 23 studies in the Asia-Pacific region. Circulation 2011;124:2056-64.

14. Hu J, Xi D, Zhao J, Luo T, Liu J, Lu H, Li M, Xiong H, Guo Z. High-density Lipoprotein and Inflammation and Its Significance to Atherosclerosis. Am J Med Sci 2016;352:408-15.

15. Lewis GF, Rader DJ. New insights into the regulation of HDL metabolism and reverse cholesterol transport. Circ Res 2005;96:1221-32.

16. Lee BC, Lee WJ, Hsu HC, Chien KL, Shih TT, Chen MF. Using clinical cardiovascular risk scores to predict coronary artery plaque severity and stenosis detected by CT coronary angiography in asymptomatic Chinese subjects. Int J Cardiovasc Imaging 2011;27:669-78.

17. Huang X, Gao S, Ma Y, Lu X, Jia Z, Hou Y. The optimal monoenergetic spectral image level of coronary computed tomography (CT) angiography on a dual-layer spectral detector CT with half-dose contrast media. Quant Imaging Med Surg 2020;10:592-603.

18. Brufau G, Groen AK, Kuipers F. Reverse cholesterol transport revisited: contribution of biliary versus intestinal cholesterol excretion. Arterioscler Thromb Vasc Biol 2011;31:1726-33.

Cite this article as: Han P, Tang J, Wang X, Su Y, Li G, Deng K. Research on the distribution spectrum of atherosclerotic plaques in patients with suspected coronary artery disease and the noninvasive screening model for coronary atherosclerosis burden. Quant Imaging Med Surg 2021;11(7):32743285. doi: 10.21037/qims-20-901
19. Shan H, Zhang M, Zhang M, Liu X, Song X, Yin X, Lv S. Association of rs5368 and rs3917406 polymorphisms in E-selectin gene with premature coronary artery disease in Chinese Han population. Int J Clin Exp Med 2015;8:4387-92.

20. Delibasi T, Karbek B, Bozkurt NC, Cakir E, Gungunes A, Ünsal ÖÖ, Aslan MS, Cakal E. Circulating E-selectin levels and insulin resistance are associated with early stages of atherosclerosis in nonfunctional adrenal incidentaloma. Arch Endocrinol Metab 2015;59:310-7.

21. Writing Group Members, Mozaffarian D, Benjamin EJ, Go AS, Arnett DK, Blaha MJ, Cushman M, Das SR, de Ferranti S, Després JP, Fullerton HJ, Howard VJ, Huffman MD, Isasi CR, Jiménez MC, Judd SE, Kissela BM, Lichtman JH, Lisabeth LD, Liu S, Mackey RH, Magid DJ, McGuire DK, Mohler ER 3rd, Moy CS, Muntner P, Mussolino ME, Nasir K, Neumar RW, Nichol G, Palaniappan L, Pandey DK, Reeves MJ, Rodriguez CJ, Rosamond W, Sorlie PD, Stein J, Towfighi A, Turan TN, Virani SS, Woo D, Yeh RW, Turner MB; American Heart Association Statistics Committee; Stroke Statistics Subcommittee. Executive Summary: Heart Disease and Stroke Statistics--2016 Update: A Report From the American Heart Association. Circulation 2016;133:447-54.

22. Emerging Risk Factors Collaboration, Di Angelantonio E, Sarwar N, Perry P, Kaptoge S, Ray KK, Thompson A, Wood AM, Lewington S, Sattar N, Packard CJ, Collins R, Thompson SG, Danesh J. Major lipids, apolipoproteins, and risk of vascular disease. JAMA 2009;302:1993-2000.

23. de Graaf MA, Broersen A, Ahmed W, Kitslaar PH, Dijkstra J, Kroft LJ, Delgado V, Bax JJ, Reiber JH, Scholte AJ. Feasibility of an automated quantitative computed tomography angiography-derived risk score for risk stratification of patients with suspected coronary artery disease. Am J Cardiol 2014;113:1947-55. 